\title{
Technical Challenges for Cognitive Radio Application in Satellite Communications
}

\author{
Daniele Tarchi*, Alessandro Guidotti*, Vincenzo Icolari*, Alessandro Vanelli-Coralli*, \\ Shree Krishna Sharma ${ }^{\dagger}$, Symeon Chatzinotas ${ }^{\dagger}$, Sina Maleki ${ }^{\dagger}$, \\ Barry Evans ${ }^{\ddagger}$, Paul Thompson ${ }^{\ddagger}$, Wuchen Tang ${ }^{\ddagger}$, Joel Grotz ${ }^{\S}$ \\ *Department of Electrical, Electronic and Information Engineering University of Bologna 40136 Bologna (Italy) \\ ${ }^{\dagger}$ SnT, University of Luxembourg L-2721 Luxembourg \\ $\ddagger$ University of Surrey, CCSR, Guildford GU2 7XH, UK \\ $\S$ Newtec Cy, Sint-Niklaas, B-9100, Belgium
}

\begin{abstract}
During the last years, spectrum scarcity has become one of the major issues for the development of new communication systems. Cognitive Radio (CR) approaches have gained an ever increasing attention from system designers and operators, as they promise a more efficient utilization of the available spectral resources. In this context, while the application of CRs in terrestrial scenarios has been widely considered from both theoretical and practical viewpoints, their exploitation in satellite communications is still a rather unexplored area. In this paper, we address the definition of several satellite communications scenarios, where cognitive radio techniques promise to introduce significant benefits, and we discuss the major enablers and the associated challenges.
\end{abstract}

\section{INTRODUCTION}

An ever increasing number of applications and services based on radio communications is rapidly consuming the limited amount of available spectral resources. The traditional fixed approach to spectrum assignments (Command \& Control) is based on the allocation of fixed portions of spectrum to specific services. This approach led to a poor spectrum utilization, as some licensed portions of the spectrum have been shown to be unused at specific times and/or in specific geographical areas. These idle spots, named spectrum holes, cannot be utilized by any other user in the current fixed spectrum allocation.

Cognitive Radio (CR) is a concept encompassing those techniques meant to improve resource usage efficiency in communication systems. In particular, CRs include all of those techniques allowing a system to access licensed spectrum frequencies on an opportunistic and dynamic basis, as long as no harmful interference to the incumbent networks [1] is produced. Thus, a CR-based system exploits spectrum awareness techniques (as spectrum sensing, databases, beamforming, etc.) in order to mitigate or avoid interference towards licensed users, which allows to opportunistically reuse temporarily or geographically available spectral resources. Although many related aspects of CRs applied to dynamic spectrum usage have been proposed and demonstrated in the literature, new solutions and application scenarios are continuously introduced. In particular, most of the academic and industrial activities have been dedicated to the design and implementation of CRs in terrestrial communications only. Very few initiatives, and mostly at academic level, have addressed CR and Satellite communications [2]-[5].

The objective of this paper is to discuss the applicability of CRs to Satellite Communications scenarios. Based on a thorough analysis of the regulatory and standardization environments, as well as of the market perspectives [6], three scenarios have been identified that can best benefit from the use of CR techniques. These scenarios have been assessed by comparing different technical enablers based on properly defined technical requirements and Quality of Service (QoS) targets. This comparison has been performed based on specific Key Performance Indicators (KPIs). It is worthwhile noting that, in this paper, we solely address the use of CR techniques in those frequencies already allocated to satellite communications. Moreover, we consider CR techniques implemented only at the satellite component side, without assuming any form of collaboration with other coexisting systems. This is due to the purpose of investigating the potential benefits of CRs when used in the already allocated spectrum bands.

The paper is organized as follows. In Section II, we introduce the identified scenarios and the proposed methodology. In Section III, technical enablers and challenges are presented and analysed, and, in Section IV, we derive our conclusions ${ }^{1}$.

\section{System Scenarios And Proposed Methodology}

In this paper, the following scenarios are considered:

- Scenario A: this scenario addresses the use of CR techniques in the downlink of a Ka-band Fixed Satellite Service (FSS) satellite system (cognitive systems) reusing frequency bands of other Broadcasting Satellite Service (BSS) Geostationary Satellite Orbit (GSO) feeder link systems (incumbent systems), Figure 1;

- Scenario B: this scenario considers the use of CR techniques in the Ka-band downlink of a FSS satellite system (cognitive system) reusing frequency bands used by Fixed

\footnotetext{
${ }^{1}$ Although the paper is mainly based on the outcomes of the activities of the European Commission funded project CoRaSat [7], the considerations expressed in this paper are solely those of the Authors and do not involve nor entail any visions of the Institutions and Companies partners of the CoRaSat consortium
} 


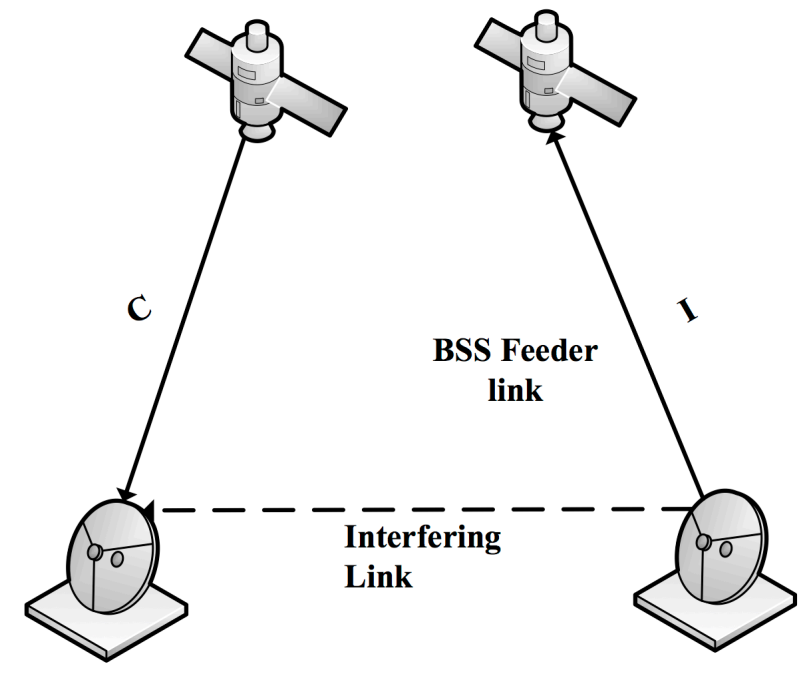

Fig. 1. Scenario A. Cognitive downlink of Ka-band FSS system with incumbent BSS feeder link. I stands for incumbent, $\mathrm{C}$ for cognitive.

Service (FS) links with priority protection (incumbent systems), Figure 2;

- Scenario C: this scenario refers to the use of CR techniques in the return link of a Ka-band FSS satellite system (cognitive system) reusing frequency bands of FS links with priority protection (incumbent systems), Figure 3.

All of these scenarios foresee the usage of non-exclusive bands allocated in secondary use cases under different conditions to satellite applications. Table I provides detailed specifications of the considered frequency bands, which are all in Ka-band [4]. It is worthwhile underlining that, in order to assess the real applicability of CRs to SatCom system, it is of paramount importance to analyze the current regulatory regime in order to identify hooks and hurdles that are to be faced when adopting $\mathrm{CR}$, and the source of interference that a cognitive satellite system may have to tackle.

This analysis has been done in [8] and [9] highlighting that, within ITU (International Telecommunication Union) region 2, the European Conference of Postal and Telecommunications Administrations (CEPT) has adopted the following decisions:

- $/ \mathrm{DEC} /(05) 08$ [10] that gives guidance on the use of the 17.3-17.7 GHz band by High Density applications in FSS (HDFSS), i.e., Scenario A;

- $\mathrm{ECC} / \mathrm{DEC} /(00) 07$ [11] that gives guidance on the use of the 17.7-19.7 GHz band by FSS and FS, i.e., Scenario B;

- ECC/DEC/(05)01 [12] that foresees that the 27.5-30.0 $\mathrm{GHz}$ band is divided between FS and FSS usage, i.e., Scenario C.

As it can be noted, these scenarios are all in the Ka-band, and this selection is the outcome of regulatory, standardization, and market analyses [8]. Ka-band is mainly used for broadband services, which are subject to market pressure for cost effective end-to-end broadband services for consumer internet access. It is also worthwhile highlighting that the ratio of the internet use is widening to $6: 1$ or higher, and thus the pressure is more

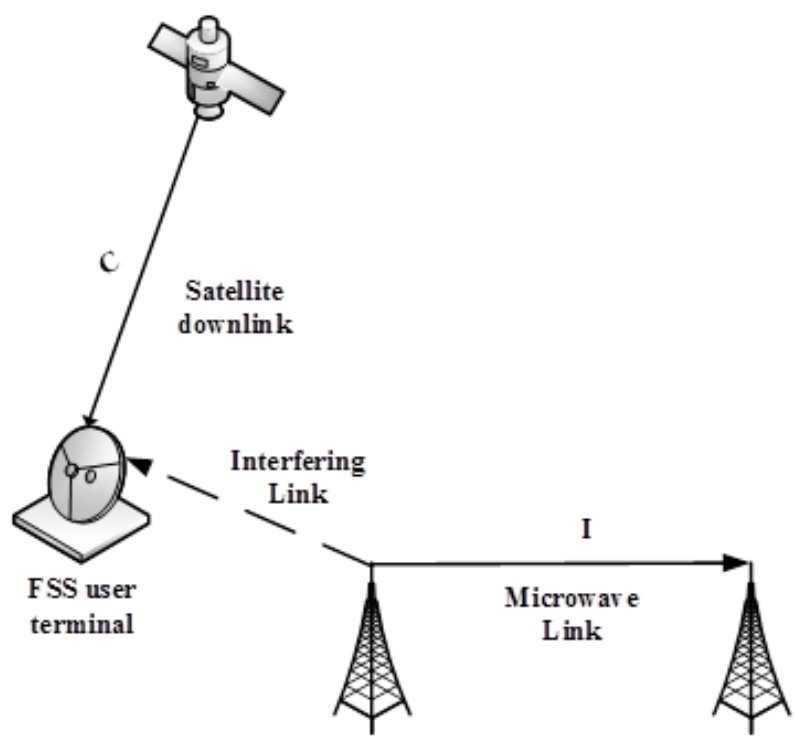

Fig. 2. Scenario B. Cognitive downlink of Ka-band FSS system with incumbent FS link. I stands for incumbent, $\mathrm{C}$ for cognitive

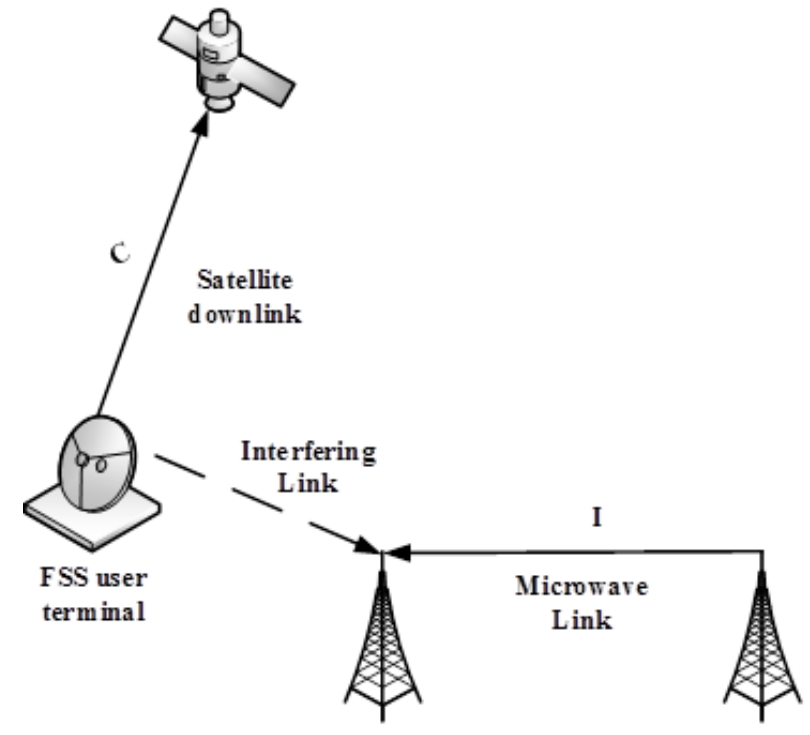

Fig. 3. Scenario C. Cognitive uplink of Ka-band FSS system with incumbent FS link. I stands for incumbent, $\mathrm{C}$ for cognitive

TABLE I

FREQUENCY BANDS - SCENARIOS OVERVIEW

\begin{tabular}{|c|c|}
\hline Scenario & Band / Usage in cognitive satellite system / Incumbent usage \\
\hline A & $\begin{array}{l}\text { 17.3-17.7GHz (Ka-band downlink) } \\
\text { Satellite downlink band to user terminals } \\
\text { Incumbent user: Satellite gateway uplinks, BSS uplinks }\end{array}$ \\
\hline B & $\begin{array}{l}\text { 17.7-19.7GHz (Ka-band downlink) } \\
\text { Satellite downlink band to FSS user terminals } \\
\text { Incumbent user: Fixed terrestrial links (microwave links) }\end{array}$ \\
\hline $\mathrm{C}$ & $\begin{array}{l}\text { 27.5-29.5GHz (Ka-band uplink, including the HDFSS band } \\
\text { 28.4465-28.9465GHz) } \\
\text { Satellite uplink band from the FSS user terminal to satellite } \\
\text { Incumbent user: Fixed services (terrestrial microwave links) }\end{array}$ \\
\hline
\end{tabular}


on the downlink. This technical path is nowadays undertaken by the majority of Satellite Operators, because Ka-band is not used so extensively, yet, by satellite broadcast services as other bands, such as Ku-band. It is also foreseen that spectrum congestions can make future Ka-band deployments more difficult, in particular at eastern orbital longitudes. As a consequence, High Throughput Satellites (HTS) are already starting to suffer from market pressure on end-to-end service costs for consumer services and also spectrum scarcity in Ka-band to deliver meaningful performance with appropriate frequency reuse schemes. This justifies the need to look for the potential application of CRs in the Ka-band satellite domain. Moreover, from a regulatory point of view, CRs have a good potential to improve sharing between the incumbent systems and cognitive satellite systems, particularly in the sharing scenarios between FS (Incumbent) versus FSS (Cognitive). In scenarios A and B there is no need for any regulatory change, but the user must be aware of the restriction of service that he might sustain if he located the FSS at a particular location. On the other hand, some modifications might be needed in the current regulations for Scenario $\mathrm{C}$.

For scenarios A and B, addressing downlink communications (Space-to-Earth), interference towards incumbent systems is basically limited (or practically excluded) by system design, since FSS terminals are receiving only. In this case, CR techniques can be used to fully exploit the available spectrum by sensing the presence of interfering FS links and mapping the geographic area of the cognitive zones. As specified in Section III-B, cognitive zones are those areas where the coexistence of incumbent and cognitive links is possible through the adoption of CR techniques. A reliable knowledge of the FS link interference might help maximizing the FSS system capacity.

In Scenario C, a sufficient protection of the FS links may be obtained by using cognitive zones around FS terrestrial link receivers in combination with FSS transmit power density limitations.

The methodology to assess these scenarios is based on the exploitation of specific enabling technologies, described in Section III. In particular, different enabling technologies are contrasted against specific technical requirements and, if they satisfy the technical requirements, evaluated on the basis of the produced improvement on several KPIs. The proposed methodology is shown in Figure 4. Satellite Manufacturers and Operators produce inputs in terms of the required system input parameters and the QoS targets. This information, combined with ITU Radiocommunication (ITU-R) and Electronic Communications Committee (ECC) regulations, leads the design of spectrum awareness and interference modelling techniques (enabling technologies), which produce an output of spectrum exploitation in terms of spectrum cartographies and noise contour floors. Once this scenario-level analysis is completed, a network management analysis is performed, in particular facing resource allocation and interference management techniques. The last step is the comparison of each of the proposed solutions for each scenarios through properly defined KPIs,

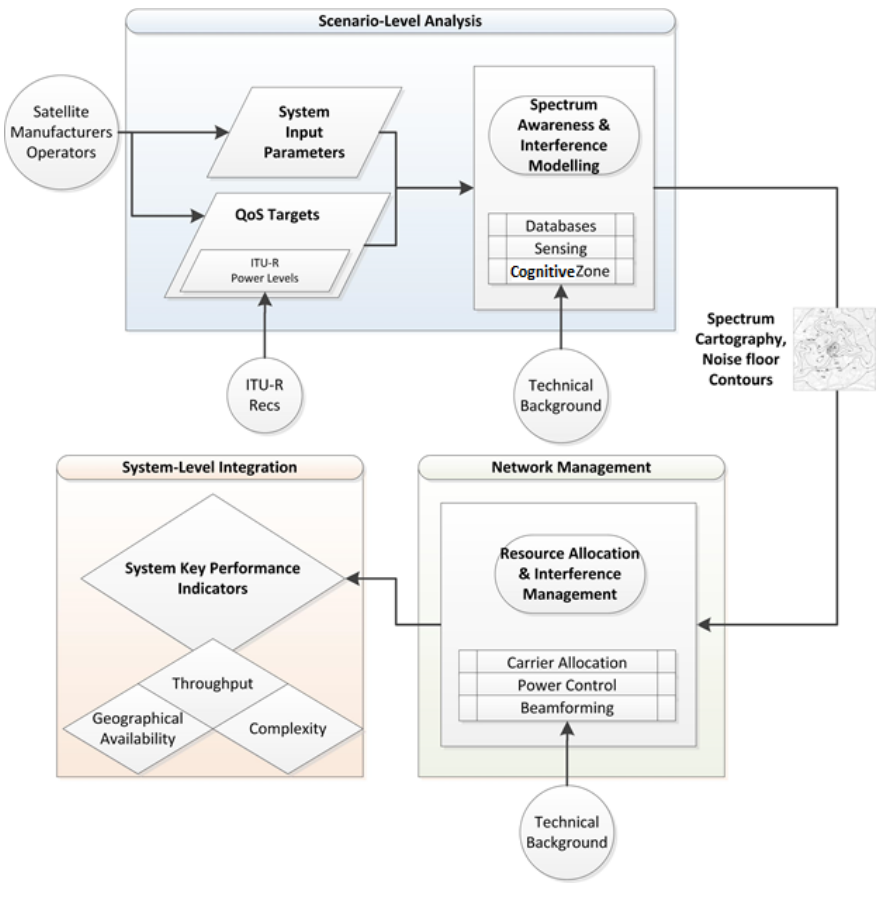

Fig. 4. The proposed assessment methodology.

which lead the selection of the most promising and feasible approaches.

\section{A. Technical Requirements and KPIs}

The aim of this subsection is to introduce the technical requirements and the KPIs that are used to evaluate the enabling technologies.

1) Technical requirements: these requirements can be classified based on the following main domains: scenario properties, QoS targets, and CR requirements.

The scenario properties category includes those constraints that are related to the specific scenario being considered. In particular, the following inputs need to be taken into account: i) the geographical parameters of the environment (e.g., coverage area, topology, etc.); ii) the device characteristics in the area of interest (e.g., location, fixed/mobile, etc.); and iii) the environment parameters (e.g., operating frequency, number of channels, etc.). The scenario properties are identified as System Input Parameters.

QoS targets targets include requirements guaranteeing a proper QoS for the cognitive communication. To this aim, the focus is both on the power levels to be respected so as to avoid the interference towards incumbent terminals and on specific parameters related to the type of service expected on the cognitive link.

As for the power levels, protection requirements for satellite and terrestrial terminals have to be considered whenever a cognitive system should be deployed on a frequency sharing basis. Some of these protection parameters are available in different ITU-R and ECC documents, which provide several recommendations for technical and operational requirements 
for the incumbent services. However, it should be noted that most of these parameters refer to terrestrial cognitive radios, and thus their potential application to satellite communications shall be carefully analyzed. Examples of such requirements are: i) protection ratios and protection margins; ii) the probability of detection; iii) Block Edge Masks (BEMs); iv) Adjacent Channel Interference Ratios (ACIRs); and v) inband and out-of-band power limits.

Concerning the service to be delivered to the cognitive user, the following requirements are considered: i) throughput; ii) availability; and iii) delay. In order to perform this servicebased evaluation, we introduce a set of reference services to be addressed in the context of the cognitive system applications. These services define the key applications used within the system and belong to one of the following categories of twoway interactive satellite systems:

- Business to consumer (B2C) applications;

- Business to business (B2B) applications;

- Machine to machine (M2M) applications.

It is worthwhile highlighting that both system input parameters and QoS targets are numerically defined by considering inputs from Satellite Manufacturers and Operators. Moreover, the definition of the power levels matching the QoS targets is based on ITU-R and ECC recommendations.

$C R$ requirements depend on the type of cognitive technique to be used:

- Database: update frequency, incumbent transmitters/receivers locations, power levels, antenna radiation patterns, polarization, etc.

- Spectrum Sensing: sensing periodicity, probability of false alarm, probability of detection, sensing thresholds, etc.

- Cognitive Zones: zone modeling and accurancy, regulatory conformance

A detailed description of these cognitive techniques is provided in Section III.

2) KPIs: the overall performance of each enabling technology is compared to the others through properly defined KPIs. Such analysis is performed for each scenario to be considered, thus allowing the identification of the best cognitive technique on a scenario-basis. The following system KPIs are considered:

- Throughput: denotes the overall capacity that the system can provide by taking into account both incumbent and cognitive systems. The system capacity is a valuable performance indicator, as the system capacity achieved through CR-based techniques is directly compared to the case of non-cognitive (exclusive) communications.

- Geographical Availability: defines the overall area where the cognitive system can be implemented subject to specific constraints. It thus compares different cognitive techniques in terms of the geographical area in which CR terminals can be deployed while satisfying specific performance requirements.
- Complexity: in order to identify the best CR technique for the specific scenario being analyzed, a complexity analysis should be performed. This in order to guarantee that the technique providing the best performance is also actually feasible based on the scenario and on the terminal capabilities.

\section{Enabling Technologies And Challenges}

Based on the previously described methodology, in this section the considered enabling techniques are presented. The aim of these CR techniques is to allow cognitive operations of the satellite links in the considered scenarios. The techniques considered in this paper are: i) spectrum databases; ii) cognitive zones; iii) spectrum sensing; and iv) beamforming. In the following, these techniques are described together with the technical challenges they pose.

\section{A. Databases}

To explore the actual characteristics of incumbent users, databases storing information on incumbent transceivers, such as Earth stations and microwave links, are required. Such databases are currently only held by national regulators and their authenticity and accuracy are highly variable. Unlike the mobile communications environment, where channel occupancy may change rapidly, in FS and FSS/BSS networks there is always a carrier present, even if it has energy dispersal imposed. One area where channel occupancy does vary is in the FSS return link, where Multiple Frequency-Time Division Multiple Access (MF-TDMA) implies a time-frequency variation of channels in the transmit band and needs further investigation. In scenarios where we need to sense the spectrum, a priori database knowledge of the channel occupancy could assist the spectrum sensing technique, increase the reactivity to spectrum changes, and save energy.

In the Ka band scenarios under investigation, databases of incumbent systems are mainly necessary to accurately evaluate the interference levels. In a specific SatCom frequency band, the information stored in databases is normally listed on a carrier-by-carrier basis within the frequency band of interest. All carriers are numbered with their frequencies and channel bandwidth, and mapped to their corresponding Earth stations. Earth stations and their corresponding satellites are both named with their individual positions (Earth station: longitude and latitude, satellite: longitude), respectively. Furthermore, parameters of the antenna for each Earth station (such as the antenna radiation pattern, elevation, azimuth, polarization, and antenna gain) are also included. Antenna radiation patterns are defined in ITU Recommendations for use in regulatory activities. The antenna polarization normally consists of linear polarization (horizontal and vertical) and circular polarization (left handed and right handed). In addition, the antenna emission is also defined by ITU classification recommendation, which is described in ITU-R SM.1138 [13]. The antenna radiation pattern for FSS is defined in ITU-R S.580 [14], while for FSS and FS the radiation patterns can be found in ITU-R S.465 [15] and ITU-R F.699 [16], respectively. 
It is wortwhile noting that proper FSS and FS databases are being made available in some countries by regulators for research activities. These databases are being used in conjunction with the ITU-R P.452 [17] propagation modelling and interference evaluation softwares to assess regions around prospective FSS sites outside of which frequency sharing can be possible. This is described in the following section as Cognitive zones.

Databases could eventually be used more operatively in the same way as in the TV White Space domain, and this is a currently ongoing activity.

\section{B. Cognitive zones}

The output provided by databases described in Section III-A is a series of contours defined by interference levels, defined as Cognitive Zones. For a specific zone being considered and for a given interference threshold, communications are allowed only with the use of a cognitive scheme aimed at reducing interference. Outside this zone, an incumbent system will not produce interference above the threshold level if the FSS is in receive mode. On the other hand, in case the FSS is transmitting, it will not produce unacceptable interference to a FS link. Cognitive zones can thus be used to ascertain acceptable geographical areas of operation for the FSS terminal. It is noted that a cognitive zone depends on the specific carrier frequency, and thus there will be a different cognitive zone for each carrier in a given band. The database incorporating the ITU-R P.452 [17] propagation models for diffraction, gaseous absorption, and rain scatter also includes terrain data and produces long- and short-term interference values which are used to plot the cognitive zones. Figures 5-6 provide an example of cognitive zones for scenarios $\mathrm{A}$ and $\mathrm{B}$, respectively. It can be seen that the area associated with a specific color represents the cognitive zone obtained based on the corresponding interference threshold level shown beside the figures.

For a proposed FSS site and carrier frequency, the cognitive zone can be calculated. Alternatively, the level of cognitive gain needed to reduce the cognitive zone to allow operation at the desired interference threshold can be computed. To assess the overall system performance, the cognitive zone approach can be used to calculate the additional geographic area that becomes available by using CR techniques, and hence the number of additional terminals. From another point of view, cognitive zones can be used to assess the interference level, which combined with link budgets allows to evaluate the overall throughput increase that can be obtained by using various levels of cognition gain.

\section{Spectrum sensing}

On the one hand, the database approach can give information about the spectrum occupancy in different bands and areas. On the other hand, cognitive zones allow the definition of area contours with a specific interfering level. Spectrum sensing can be considered as the low level operation for having a current spectrum awareness in a certain position. In fact,

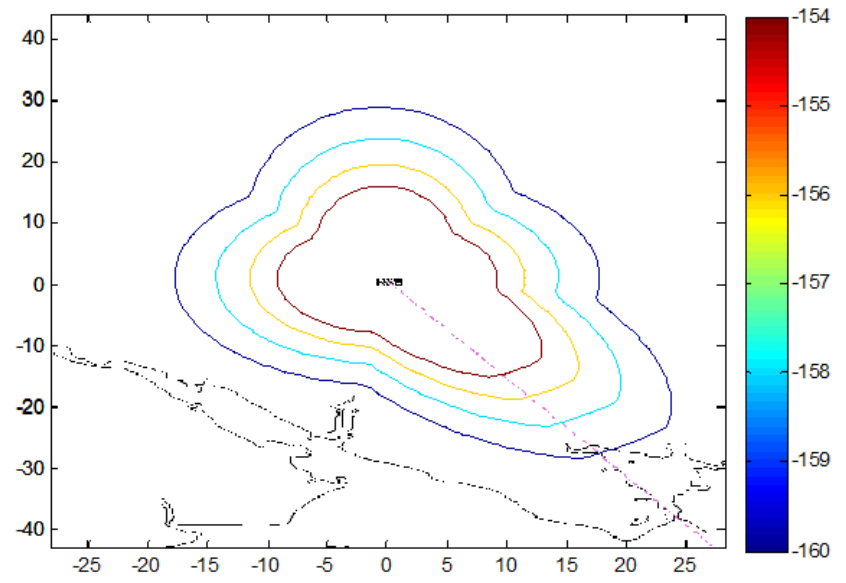

Fig. 5. Example of Cognitive Zone for Scenario A with interference from BSS to FSS (Axes are in km from the BSS uplink transmitter; contours are for interference level in $\mathrm{dBW} / \mathrm{MHz}$ ).

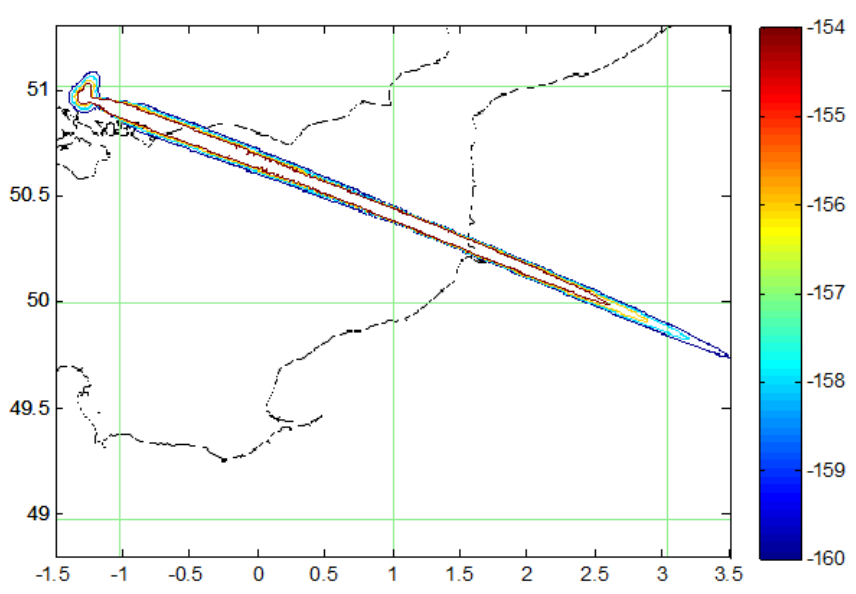

Fig. 6. Example of Cognitive Zone for Scenario B with interference from FS to FSS (Axes are latitude and longitude; contours are for interference level in $\mathrm{dBW} / \mathrm{MHz})$

differently from databases information that need to be updated, the cognitive system can acquire more recent information about spectrum availability thanks to spectrum sensing [18].

Hence, the aim of spectrum sensing is the detection of incumbent user signals by scanning some selected frequency bands. Several approaches are possible for spectrum sensing, and all refer to the detection of an unknown or partially known signal. The efficiency of a spectrum sensing technique can thus be measured as a trade-off between the probability of false alarm and the probability of detection (or misdetection) for achieving a proper degree of certainty in its detection.

In particular, when a misdetection occurs, in scenarios A and $\mathrm{B}$ a damage to the cognitive receivers is introduced, while in Scenario C the incumbent user would be harmed. Several 


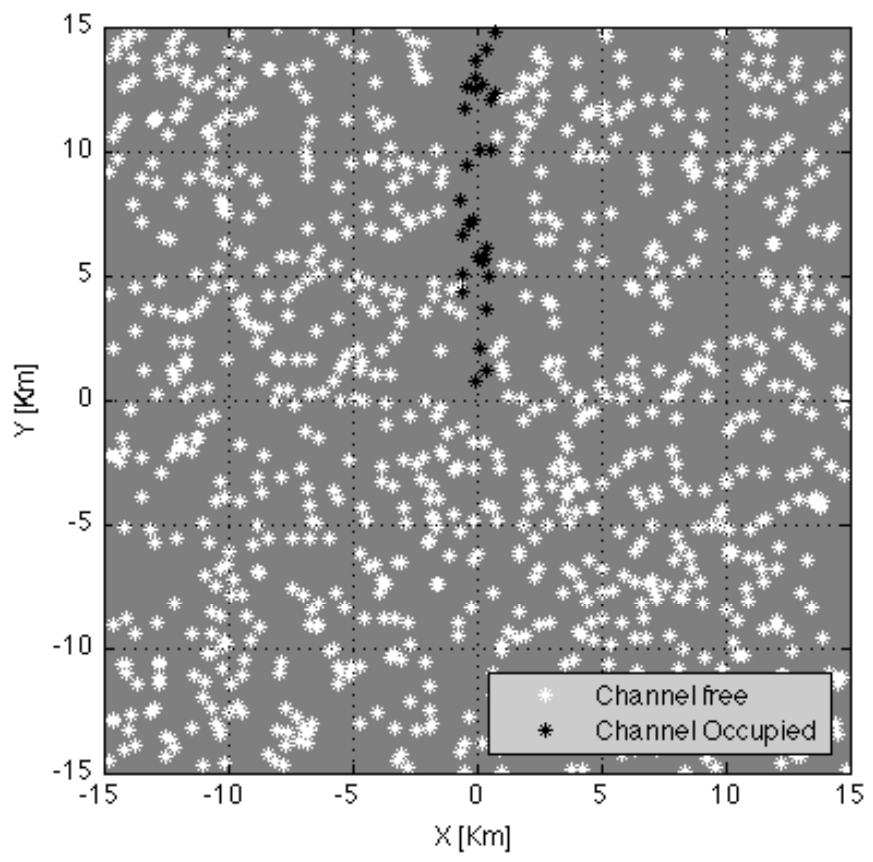

Fig. 7. Geographical representation of Energy Detector effectiveness in an area with an FS incumbent link (scenario B).

factors affect the sensing performance: Sensing Time, Channel Characteristics, Signal-to-noise Ratio (SNR). However, especially for Scenarios A and B where spectrum sensing seems to be more feasible, the cognitive terminal should implement a spectrum sensing technique to assess the presence of an incumbent user.

Despite the manifold detection algorithms that the literature presents, we refer to Energy Detection and Cyclostationary Feature Detection algorithms [18], as they are the most promising and considered in a cognitive environment.

An Energy Detector aims at detecting the presence of an incumbent system by estimating the received power in a given bandwidth. The received power is then compared to a specific threshold, so that if the detected energy is above the threshold, the detector decides that a signal is present. In Figure 7, the geographical representation of the effectiveness of the Energy Detector in the Scenario B is reported, where the detected incumbent signals in different locations can be seen.

A cyclostationary Feature Detection algorithm is based on the detection of a cyclostationary pattern in the incumbent signal. This property can be exploited by focusing on the incumbent signal structure. Indeed, a cyclostationary algorithm allows to detect the presence of a repeated pattern, such as a certain modulation scheme in the incumbent system. This property is not present in case of presence of noise only. A cyclostationary algorithm is less conditioned by the noise power, but it needs to know some specific properties on the signal to be detected in terms of signal pattern.

\section{Beamforming}

In the previously described scenarios, beamforming can be applied as follows: i) beamforming for Spectrum Sensing (SS)/interference detection; and ii) beamforming for improving the Signal-to-Interference plus Noise Ratio (SINR) of the satellite terminals.

1) Beamforming for SS: In scenario B, the main problem is the detection of harmful FS signals in order to protect the FSS terminals. A receiver beamformer can be designed in order to detect the harmful FS signals so that the satellite terminal can avoid them. In this context, the possibility of putting an omnidirectional antenna (dipole) in addition to the existing satellite dish has been studied in [19]. Although it is possible to perform sensing using existing satellite dishes, it requires the cancellation of DVB-S2 (Digital Video Broadcasting Satellite - Second Generation) signals and thus the receiver operates at lower SNRs as compared to the dipole reception. Considering the long-term interference criteria, the I/N target of $-10 \mathrm{~dB}$ can be considered as the tolerable interference threshold for protecting FSS terminal [20]. Another solution to the considered coexistence problem is the joint processing of the signals received by two antennas in order to extract the desired FSS signal from the total received signal. For this purpose, suitable angular beamforming approaches such as Linearly Constrained Minimum Variance (LCMV), and Minimum Variance Distortionless Response (MVDR) [21], [22] can be applied based on the Direction of Arrival (DoA) information of the FS transmitters available from the database. On the other hand, the beamformer can also be used to enhance the sensing of harmful FS signals by focusing its receiving beam in the direction of the FS transmitter. For the above applications, the effect of antenna generated power imbalance has to be investigated in detail since dipole and dish antennas equipped in the FSS terminal receive two signals of different strengths.

2) Beamforming for Interference Management: In the selected scenarios, beamforming can be employed at the terminal side as:

a) Multi Low Noise Block (LNB) at the Terminal-side:

The MultiLNB-based beamforming can be applied in scenarios B and C. In scenario B, a satellite terminal equipped with multiple LNBs can be used as a receive beamformer while in scenario $\mathrm{C}$, it can be used as a transmit beamformer. In practice, the number of LNBs should be kept low, e.g., 2-3 LNBs, due to cost, mechanical support and electromagnetic blockage issues [23]. It can be noted that, in the presence of multiple harmful FS links, the considered scenario becomes overloaded since the satellite receiver usually has fewer LNBs than the received co-channel FS signals. In this context, a overloaded receiver structure with $M$ number of LNBs has been proposed in [23] for broadcast reception purpose. Similar concept can be applied in order to improve the detection of DVB-S2 signal reception in the presence of multiple harmful FS interfering users. The main difference in the considered scenario from the overloaded scenario considered in [23] is 
that the FS interference can enter to the FSS satellite terminal's receiving pattern from any direction instead of the main lobe.

\section{CONCLUSIONS}

The exploitation of congitive radio techniques in SatCom environments is gaining an increased importance from both market and technical viewpoints. In this paper, after the definition of possible scenarios for the CR usage in SatComs, the evaluation of advantages of implementing CR techniques in SatComs systems as a way for increasing the spectrum utilization considering the regulatory, standardization, and market frameworks is given. To this aim, the most attractive technical enablers have been identified: database, cognitive zones, spectrum sensing and beamforming. The main oucomes of the performed study on the four enablers is that: (i) databases, allowing to have knowledge of the surrounding environment by exploiting the presence of stored information about the incumbent users, can be used for assisting spectrum sensing techniques, increase the CR system reactivity and save energy; (ii) the cognitive zones, by exploiting the databases, allow to define the areas where the interference level overcomes a certain value. The knowledge of cognitive zones allows to know where CR techniques should be used for incraesing the system performance in terms of geographical availability; (iii) the spectrum sensing tecniques, allowing to sense the presence of incumbent users, are proposed as a solution for detecting the presence of FS systems in a blind way. This can occur whenever no information of their presence is given by a database; in particular energy detector and cyclostationary feature detectors have been considered, allowing to have quite good performance in selected scenarios; (iv) beamforming techniques can be used as a way for improving the spectrum sensing but also for mitigating the interference between incumbent and cognitive users, by exploiting the beam direction toward the incumbent in the sensing node for improving the spectrum sensing, or by limiting the harmful interference from the incumbent, by opportunely directing the cognitive terminal beams.

\section{ACKNOWLEDGMENT}

The authors would like to thank the European Commission (EC) for their co-funding support through the CoRaSat project (FP7 ICT STREP Grant Agreement n. 316779).

\section{REFERENCES}

[1] I. F. Akyildiz, W.-Y. Lee, M. C. Vuran, and S. Mohanty, "Next generation/dynamic spectrum access/cognitive radio wireless networks: A survey," Computer Networks, vol. 50, no. 13, pp. 2127 - 2159, 2006. [Online]. Available: http://www.sciencedirect.com/science/article/ pii/S1389128606001009

[2] R. Suffritti, G. E. Corazza, A. Guidotti, V. Petrini, D. Tarchi, A. Vanelli-Coralli, and M. Di Renzo, "Cognitive hybrid satelliteterrestrial systems," in Proceedings of the 4th International Conference on Cognitive Radio and Advanced Spectrum Management, ser. CogART '11. New York, NY, USA: ACM, 2011, pp. 64:1-64:5. [Online]. Available: http://doi.acm.org/10.1145/2093256.2093320

[3] S. K. Sharma, S. Chatzinotas, and B. Ottersten, "Satellite cognitive communications: Interference modeling and techniques selection," in 6th Advanced Satellite Multimedia Systems Conference (ASMS) and 12th Signal Processing for Space Communications Workshop (SPSC), Sept 2012, pp. 111-118.
[4] K. Liolis, G. Schlueter, J. Krause, F. Zimmer, L. Combelles, J. Grotz, S. Chatzinotas, B. Evans, A. Guidotti, D. Tarchi, and A. Vanelli-Coralli, "Cognitive radio scenarios for satellite communications: The corasat approach," in Future Network and Mobile Summit (FutureNetworkSummit), 2013, July 2013, pp. 1-10.

[5] S. K. Sharma, S. Chatzinotas, and B. Ottersten, "Cognitive radio techniques for satellite communication systems," in IEEE 78th Vehicular Technology Conference (VTC Fal), Sept 2013, pp. 1-5.

[6] CoRaSat, "Deliverable D2.1, Service and Market Requirements," 2013. [Online]. Available: http://www.ict-corasat.eu/documents/deliverables

[7] (2014) CoRaSat, Cognitive Radio for Satellite Communiations. [Online]. Available: http://www.ict-corasat.eu/

[8] CoRaSat, "Deliverable D2.2, Regulatory, Standardisation and Technology Framework," 2013. [Online]. Available: http://www. ict-corasat.eu/documents/deliverables

[9] — - "Deliverable D2.3, Scenarios Definition and Selection," 2013. [Online]. Available: http://www.ict-corasat.eu/documents/deliverables

[10] CEPT, "Ecc decision: The availability of frequency bands for high density applications in the fixed-satellite service (space-to-earth and earth-to-space)," CEPT, Tech. Rep., 2013.

[11] — "Erc decision: on the shared use of the band 17.7 - $19.7 \mathrm{ghz}$ by the fixed service and earth stations of the fixed-satellite service (spaceto-earth)," CEPT, Tech. Rep., 2000.

[12] — "The use of the band 27.5-29.5 ghz by the fixed service and uncoordinated earth stations of the fixed-satellite service (earth-tospace)," CEPT, Tech. Rep., 2013.

[13] ITU-R, "Recommendation itu-r sm.1138: Determination of necessary bandwidths including examples for their calculation and associated examples for the designation of emissions," ITU, Tech. Rep., 2008.

[14] — - "Recommendation itu-r s.580: Radiation diagrams for use as design objectives for antennas of earth stations operating with geostationary satellites," ITU, Tech. Rep., 2003.

[15] — , "Recommendation itu-r s.465: Reference earth-station radiation pattern for use in coordination and interference assessment in the frequency range from 2 to about 30 ghz," ITU, Tech. Rep., 1993.

[16] _ "Recommendation itu-r f.699: Reference radiation patterns for fixed wireless system antennas for use in coordination studies and interference assessment in the frequency range from $100 \mathrm{mhz}$ to about 70 ghz," ITU, Tech. Rep., 2004.

[17] _ "Recommendation itu-r p.452: Prediction procedure for the evaluation of microwave interference between stations on the surface of the earth at frequencies above about 0.7 ghz*," ITU, Tech. Rep., 2003.

[18] T. Yucek and H. Arslan, "A survey of spectrum sensing algorithms for cognitive radio applications," IEEE Communications Surveys Tutorials, vol. 11, no. 1, First 2009

[19] S. K. Sharma, S. Maleki, S. Chatzintoas, J. Grotz, and B. ottersten, "Implementation aspects of cognitive techniques for ka band (17.7-19.7 ghz) satcoms," in submitted to 7th ASMS/13th SPSC, 2014.

[20] ITU-R, "Recommendation itu-r f.758: Considerations in the development of criteria for sharing between the terrestrial fixed service and other services," ITU, Tech. Rep., 2003.

[21] S. K. Sharma, S. Chatzinotas, and B. Ottersten, "Spatial filtering for underlay cognitive satcoms," in 5th Int. Conf. PSATS, June 2013, pp. $186-198$.

[22] —, "Transmit beamforming for spectral coexistence of satellite and terrestrial networks," in 8th Int. Conf. CROWNCOM, July 2013, pp. $275-281$.

[23] J. Grotz, B. Ottersten, and J. Krause, "Signal detection and synchronization for interference overloaded satellite broadcast reception," IEEE Trans. Wireless Commun., vol. 9, no. 10, pp. 3052-3063, October 2010. 